\title{
Regulation of Dendrobium Polysaccharides on Proliferation and Oxidative Stress of Human Umbilical Vein Endothelial Cells in the High Glucose Environment
}

\author{
Yajia Li $\mathbb{D},{ }^{1,2,3}$ Ziqin Cao $\mathbb{D}^{4},{ }^{4}$ Limin Jia, ${ }^{2,3}$ Yanfei Huang, ${ }^{2,3}$ Meilan Shi $\mathbb{D}^{2,3,5}$ \\ and Qiangxiang $\mathrm{Li} \mathbb{1}^{2,3,6}$ \\ ${ }^{1}$ Department of Dermatology, Xiangya Hospital, Central South University, Changsha, China \\ ${ }^{2}$ National Clinical Research Center for Geriatric Disorders, Xiangya Hospital, Central South University, Changsha, Hunan, China \\ ${ }^{3}$ Ningxia Geriatric Disease Clinical Research Center, People's Hospital of Ningxia Hui Autonomous Region, Yinchuan, \\ Ningxia Hui Autonomous Region, China \\ ${ }^{4}$ Department of Spine Surgery, The Xiangya Hospital, Central South University, Changsha, Hunan, China \\ ${ }^{5}$ Loudi Central Hospital, the Loudi Affiliated Hospital of Nanhua University, Hunan province, Loudi, 417000, China \\ ${ }^{6}$ Hunan People's Hospital, Department of Hunan Institute of Geriatrics, Changsha, China
}

Correspondence should be addressed to Meilan Shi; xiaoxiannvjiafei@163.com and Qiangxiang Li; liqiangxiang@nxmu.edu.cn

Received 15 October 2020; Revised 2 May 2021; Accepted 31 May 2021; Published 14 June 2021

Academic Editor: Almudena G mez Hern ndez

Copyright (C) 2021 Yajia Li et al. This is an open access article distributed under the Creative Commons Attribution License, which permits unrestricted use, distribution, and reproduction in any medium, provided the original work is properly cited.

\begin{abstract}
Backgrounds. Polysaccharides of Dendrobium candidum (PDC) showed a strong antioxidant effect on islet cells while the effects of PDC on human umbilical vein endothelial cells (HUVECs) under the high glucose condition remain unclear. Material and Method. HUVECs were incubated with high glucose $(33.3 \mathrm{mmol} / \mathrm{L})$ for 48 hours to induce injury, and cells were treated with PDC (100, 200, and $400 \mu \mathrm{g} / \mathrm{mL}$ ) for 48 hours. The tetrazolium blue colorimetric (MTT) assay was used to detect cell proliferation, superoxide dismutase (SOD), and nitric oxide (NO) content in cell supernatants. Flow cytometry was used to detect reactive oxygen species (ROS) and calcium levels. Results. (1) Compared with the control group, the proliferation of HUVECs cells in the high glucose $(33.3 \mathrm{mmol} / \mathrm{L})$ group decreased $(P<0.05)$. The intracellular calcium ion concentration and the intracellular ROS level increased $(P<0.01$ and $P<0.05)$. SOD activity and the level of NO in the culture medium were reduced $(P<0.05)$. $(2)$ Compared with the control group, PDC $(50,100,200,400$, and $800 \mu \mathrm{g} / \mathrm{mL})$ did not significantly affect the cell proliferation of HUVECs $(P>0.05)$. (3) Compared with the high glucose group, the HUVEC cell viability of the high glucose + PDC (100, 200, and 400 $\mu \mathrm{g} / \mathrm{mL})$ group increased while the intracellular calcium ion concentration decreased in a concentration-dependent manner $(P<0.05)$. Intracellular ROS levels were reduced, while SOD activity and the level of NO in culture fluids increased $(P<0.05)$. Conclusion. PDC can promote the proliferation of HUVECs in the high glucose environment by reducing oxidative stress injury of HUVECs induced by high glucose.
\end{abstract}

\section{Background}

The major chronic complications of diabetes (diabetes mellitus, DM) involve the diseases of heart, eye, kidney, foot, and neuropathy, all related to vascular injury $[1,2]$. Many researchers have focused on diabetic vascular disease but the pathogenesis of diabetic vascular disease has yet to be further clarified. Increased oxidative stress is one of the patho- genesis of diabetic microvascular complications. Its role in the chronic pathogenesis of diabetes is not only direct cytotoxic damage but also can act as an important intracellular messenger to activate signaling pathways, directly leading to tissue and cell damage [3,4]. Previous studies have confirmed that increased reactive oxygen species (ROS) production can lead to decreased endothelial cell survival, and the induction of endothelial cell damage is related to increased 
intracellular free calcium and activation of $\operatorname{ROS}[1,5,6]$. Intracellular levels of reactive ROS and free calcium can contribute to dysfunction and progressive loss of beta cells and thereby to diabetes mellitus. Besides, as an antioxidant enzyme in mitochondria, superoxide dismutase (SOD) plays an important role in combating oxidative damage. The decrease of SOD activity is an important biochemical characteristic of diabetic patients. Decreased endotheliumdependent vasodilation in diabetes may be related to both the reduced nitric oxide (NO) synthesis and increased $\mathrm{NO}$ inactivation $[7,8]$. Dendrobium polysaccharide, also called polysaccharides of Dendrobium candidum (PDC), is the main active ingredient in Dendrobium candidum [9]. Studies have shown that PDC has a strong antioxidant effect in vitro, which can inhibit islet cell apoptosis and necrosis, protect islet cells, resist calcium overload, and protect and repair cells [10-12].

Therefore, we speculated that PDC might regulate the oxidative stress of human umbilical vein endothelial cells (HUVECs) under high glucose environment, promote the proliferation of HUVECs, and thus exert its effect on protecting endothelial cells. This study was to explore the effects of PDC on HUVECs by detecting the SOD activity and NO content, as well as the ROS and calcium levels in high glucose conditions.

\section{Materials and Methods}

2.1. Experimental Materials. HUVECs were purchased from the Institute of Pharmacology of Central South University; active oxygen detection kit, Fluo-3AM (calcium ion fluorescence probe), was purchased from (Jiangsu Province Biyuntian Institute of Biotechnology); PDC was prepared by this research group; low glucose DMEM culture solution was purchased from (Gibco, USA); fetal bovine serum was purchased from (APP, Australia); D-glucose and trypsin powder was purchased from (Sigma, USA); $\mathrm{CO} 2$ culture box was purchased from (Shelodn company); inverted biological microscope was purchased from (Japan OLYMPUS company); automatic microplate reader was purchased from (Japan CORONA company); LDZX-40C vertical self-control electric heating pressure steam sterilizer was purchased from (Shanghai Shenan Medical Instrument Factory); KH $-600 \mathrm{DB}$ data ultrasonic cleaner was purchased from (Kunshan Hechuang Ultrasonic Instrument Co., Ltd.); flow cytometer was purchased from (BECTON DICKINSON Company, USA).

\subsection{Establishment of the High Glucose Model and} Experimental Grouping after Cell Culture and Generation of Umbilical Vein Endothelial Cells. When the cells reach 80\% confluence, discard the serum-containing culture medium, change the serum-free medium for 24 hours (h), and then change to a culture medium with different concentrations of glucose, which are $17.5 \mathrm{mmol} / \mathrm{L}$ and $25 \mathrm{mmol} / \mathrm{L}$, and $33.3 \mathrm{mmol} / \mathrm{L}, 40 \mathrm{mmol} / \mathrm{L}$, and $56 \mathrm{mmol} / \mathrm{L}$. After observation at $12,24,36,48$, and $72 \mathrm{~h}$, the optimal glucose concentration for establishing a high glucose model of umbilical vein endothelial cells was $33 \mathrm{mmol} / \mathrm{L}$.

\subsection{Experimental Grouping}

2.3.1. Effect of Different Concentrations of PDC on the Growth of HUVECs. The study was done on HUVECs treated for 48 hours in different groups: one control group and other groups with different concentrations of PDC $(50,100,200$, 400 , and $800 \mu \mathrm{g} / \mathrm{mL}$ ).

2.3.2. Effects of Various Glucose Concentrations on the Proliferation of HUVECs. The study was done on HUVECs treated with glucose for 48 hours in different groups: one control group and other groups with different concentrations of glucose $(17.5 \mathrm{mmol} / \mathrm{L}, 25 \mathrm{mmol} / \mathrm{L}, \quad 33.3 \mathrm{mmol} / \mathrm{L}, 40$ $\mathrm{mmol} / \mathrm{L}$, and $56 \mathrm{mmol} / \mathrm{L}$ ).

2.3.3. Effect of PDC on the Growth of HUVECs under High Glucose Environment In Vitro. The study was done on cell treated for 48 hours in groups with high concentration like high glucose group $(33.3 \mathrm{mmol} / \mathrm{L})$ and high glucose + different concentrations of $\operatorname{PDC}(100,200$, and $400 \mu \mathrm{g} / \mathrm{mL})$ compared to the control group.

2.3.4. Effect of PDC on HUVEC Active Oxygen and Intracellular Calcium Ion Concentration, Superoxide Dismutase Activity, and Nitric Oxide Content in Cell Supernatant under the High Glucose Environment In Vitro. The experiment was divided into a control group, a high glucose group $(33.3 \mathrm{mmol} / \mathrm{L})$, and a high glucose + PDC (100, 200 , and $400 \mu \mathrm{g} / \mathrm{mL}$ ) cotreatment group. The treatment time was 48 hours. Set five replicates in each group and repeated three times.

2.4. Tetrazolium Blue Colorimetric (MTT) Method Was Used to Detect Cell SOD and Nitric Oxide (NO) Content. HUVEC was washed with PBS and digested, and cell suspension was made with the M200 medium containing $0.5 \%$ FBS. After counting the cells, seed is $5 \times 10^{3} / 150 \mu \mathrm{L}$ per well in a 96well plate. The whole medium was cultured for 24 hours, the culture medium was discarded, and the medium was only pretreated with $0.5 \%$ FBS for 24 hours to achieve cell synchronization. Add the control group, experimental group, and positive control medium. After continuing the normal culture for $12,24,36,48$, and 72 hours, $50 \mu \mathrm{L}$ of MTT solution was added, and the cell proliferation was measured by the microplate reader using the MTT method at $490 \mathrm{~nm}$ and $690 \mathrm{~nm}$ (OD) of each well. Set 3 replicates in each group and repeat the experiment at least three times. Measurement of NO: set up a blank tube (no sample and sodium nitrite added), a standard tube (add $20 \mu \mathrm{mol} / \mathrm{L}$ sodium nitrite), and a determination tube (add $0.3 \mathrm{~mL}$ of the supernatant to be tested). The tubes were mixed and placed for 10 minutes, centrifuged at 3500 4000 r/min for 10 minutes, and $0.8 \mathrm{~mL}$ of the supernatant was taken. Add $0.4 \mathrm{~mL}$ of developer and mix well and measure the absorbance after 15 minutes. Find the corresponding NO concentration in the standard curve (sodium nitrite series solution) prepared in advance. Absorbance of $\mathrm{NO}$ was measured at $540 \mathrm{~nm}$; measurement of SOD: set up a test tube and a control tube according to the requirements of the test box and add various reaction solutions and the supernatant to be tested to the test tube and 
mix well. Set the temperature constantly of the water bath to $37^{\circ} \mathrm{C}$ for $40 \mathrm{~min}$, add the color reagent and mix well, pour it into a $1 \mathrm{~cm}$ optical path cuvette after $10 \mathrm{~min}$, adjust to zero with distilled water, and compare the color with a microplate reader at a wave length of $550 \mathrm{~nm}$. According to the formula SOD activity (U/mgprot) $=[$ control tube - measurement tube)/control tube $] \div 50 \% \times$ (total volume of reaction solution /sampling amount $) \div$ protein content in tissue $(\mathrm{mgprot} / \mathrm{mL})$, to calculate SOD activity, no clear sample is added to the control tube, and the others are the same as the measurement tube [13-15].

2.5. Detection of ROS and Calcium Ion by Flow Cytometry. The cells over $75 \mathrm{ml}$ bottles were evenly seeded on a sixwell plate, and PDC $(100,200,400 \mu \mathrm{g} / \mathrm{ml})$ was added for 48 h. Each group was set up with 5 duplicate wells and repeated three times; the culture solution was aspirated. Each group contains three parallel samples. Detection and analysis by flow cytometry. The recommended excitation wavelength is $488 \mathrm{~nm}$, and the emission wavelength is $525-530 \mathrm{~nm}$. Reactive Oxygen Species Assay Kit is one of the most commonly used methods to quantitatively detect the level of reactive oxygen species in cells by the change in fluorescence intensity of the fluorescent dye DCFH-DA. Fluo-3 AM is a fluorescent dye that penetrates cell membranes. Fluo- 3 can be combined with calcium ions, which can produce strong fluorescence when combined with calcium ions. The maximum excitation wavelength is $506 \mathrm{~nm}$, and the maximum emission wavelength is $526 \mathrm{~nm}$.

2.6. Data Statistics and Analysis. All data in the experiment are expressed as mean \pm standard deviation $(\bar{x} \pm s)$, and statistical processing is performed using international general statistical software SPSS16.0. The experimental data were analyzed by one-way ANOVA, and the pairwise comparison of different groups was performed by the SNK-q test. $P<$ 0.05 was considered significant.

\section{Results}

3.1. Effect of PDC or High Glucose In Vitro on HUVEC Proliferation. As shown in Table 1, according to the results of MTT, compared with the control group, PDC at 50, 100, 200,400 , and $800 \mu \mathrm{g} / \mathrm{mL}$ did not significantly inhibit HUVECs $(P>0.05)$. Therefore, it can be judged that PDC has no obvious cytotoxic effect. Further, explore the effects of different glucose concentrations on the proliferation of HUVECs, as shown in Table 2: compared with the control group (OD value was $1.09 \pm 0.14$ ), the concentration of $25.5,33.3,40.0$, and $56.0 \mathrm{mmol} / \mathrm{L}$ glucose significantly inhibited HUVEC cell proliferation with OD values of $0.75 \pm 0.09$, $0.42 \pm 0.05,0.31 \pm 0.04$, and $0.19 \pm 0.03$, respectively, which also showed a concentration dependence $(P<0.05)$. When the concentration reached $33.3 \mathrm{mmol} / \mathrm{L}$, the inhibition rate of HUVEC cell proliferation was $51.65 \%$, which was the optimal glucose concentration in the culture medium. However, the cell proliferation ability of the mannitol $(56.0 \mathrm{mmol} / \mathrm{L})$ group was not significantly different from that of the control group $(P>0.05)$, indicating that high glucose caused reduced
TABLE 1: Effects of different concentrations of Dendrobium candidum polysaccharides (PDC) on the proliferation of HUVEC cells.

\begin{tabular}{lc}
\hline Group & OD value \\
\hline Control group & $1.17 \pm 0.16$ \\
PDC $(50 \mu \mathrm{g} / \mathrm{mL})$ & $1.08 \pm 0.12$ \\
PDC $(100 \mu \mathrm{g} / \mathrm{mL})$ & $1.09 \pm 0.09$ \\
PDC $(200 \mu \mathrm{g} / \mathrm{mL})$ & $1.13 \pm 0.16$ \\
PDC $(400 \mu \mathrm{g} / \mathrm{mL})$ & $1.08 \pm 0.17$ \\
PDC $(800 \mu \mathrm{g} / \mathrm{mL})$ & $1.11 \pm 0.12$ \\
\hline
\end{tabular}

Note: HUVECs were incubated with PDC $(50,100,200,400$, and $800 \mu \mathrm{g} / \mathrm{mL})$ for 48 hours. The MTT method was used to detect cell proliferation. All data are expressed as $\bar{x} \pm S(n=5)$.

TABLE 2: Effects of different glucose concentrations on HUVEC cell proliferation.

\begin{tabular}{lc}
\hline Group & OD value \\
\hline Control & $1.09 \pm 0.14$ \\
High glucose $(17.5 \mathrm{mmol} / \mathrm{L})$ & $1.01 \pm 0.12$ \\
High glucose $(25.5 \mathrm{mmol} / \mathrm{L})$ & $0.75 \pm 0.09^{*}$ \\
High glucose $(33.3 \mathrm{mmol} / \mathrm{L})$ & $0.42 \pm 0.05^{*}$ \\
High glucose $(40.0 \mathrm{mmol} / \mathrm{L})$ & $0.31 \pm 0.04^{* *}$ \\
High glucose $(56.0 \mathrm{mmol} / \mathrm{L})$ & $0.19 \pm 0.03^{* *}$ \\
Mannitol $(56.0 \mathrm{mmol} / \mathrm{L})$ & $1.11 \pm 0.13$ \\
\hline
\end{tabular}

Note: HUVECs were incubated with D-glucose $(17.5,25.5,33.3,40.0$, and $56.0 \mathrm{mmol} / \mathrm{L})$ and mannitol $(56.0 \mathrm{mmol} / \mathrm{L})$ for 48 hours. The MTT method was used to detect cell proliferation. All data are expressed as $\overline{x \pm S}(n=5)$. ${ }^{*} P<0.05,{ }^{* *} P<0.01$, compared with the control group.

TABle 3: Effects of different concentrations of Dendrobium candidum polysaccharides (PDC) on the proliferation of HUVEC cells cultured in high glucose.

\begin{tabular}{lc}
\hline Group & OD value \\
\hline Control & $1.29 \pm 0.10$ \\
High glucose $(33.3 \mathrm{mmol} / \mathrm{L})$ & $0.39 \pm 0.03^{* *}$ \\
High glucose + PDC $(100 \mu \mathrm{g} / \mathrm{mL})$ & $0.50 \pm 0.05^{\#}$ \\
High glucose + PDC $(200 \mu \mathrm{g} / \mathrm{mL})$ & $0.73 \pm 0.07^{\#}$ \\
High glucose + PDC $(400 \mu \mathrm{g} / \mathrm{mL})$ & $0.89 \pm 0.05^{\# \#}$ \\
\hline
\end{tabular}

Note: both D-glucose $(33.3 \mathrm{mmol} / \mathrm{L})$ and PDC $(100,200$, and $400 \mu \mathrm{g} / \mathrm{mL})$ were incubated with HUVECs for 48 hours. The MTT method was used to detect cell viability. All data are expressed as $\overline{x \pm S}(n=5) .{ }^{* *} P<0.05$, compared with control group; ${ }^{\#} P<0.05,{ }^{\# \#} P<0.05$ compared with the high glucose group.

HUVEC cell proliferation, and induced cell damage was not related to osmotic pressure. The results are shown in Table 3: compared with the high glucose group (OD value was $0.43 \pm 0.05)$, the cell proliferation of the high glucose + PDC $(100,200$, and $400 \mu \mathrm{g} / \mathrm{mL})$ group significantly increased (OD values were $0.58 \pm 0.06,0.78 \pm 0.08$, and $0.92 \pm 0.08$, 


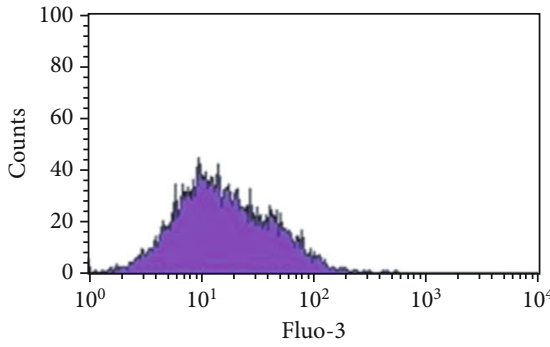

$\mathrm{C}$

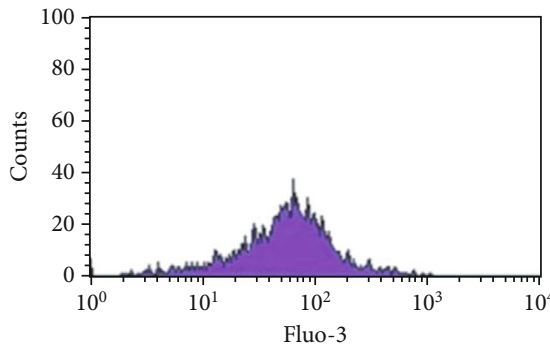

HG

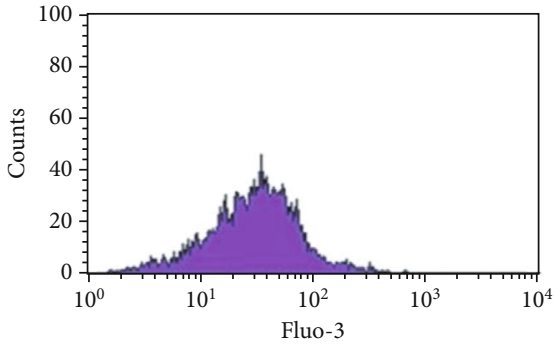

$\operatorname{PDC}(100 \mu \mathrm{g} / \mathrm{mL})$

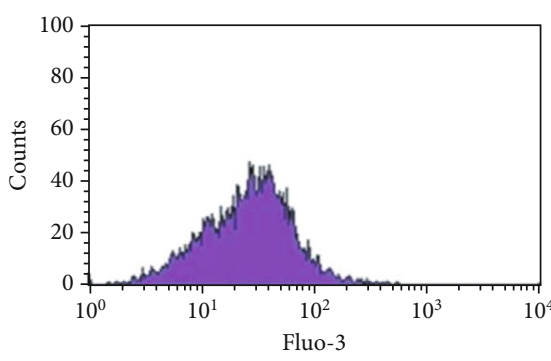

$\operatorname{PDC}(200 \mu \mathrm{g} / \mathrm{mL})$

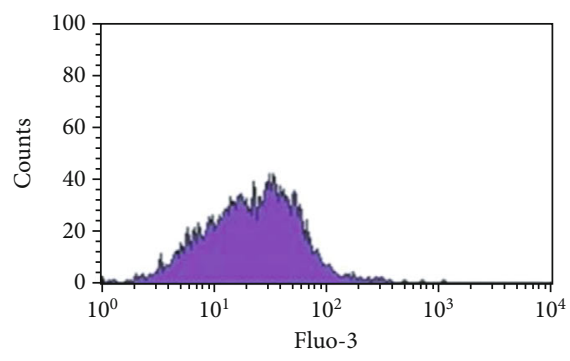

$\operatorname{PDC}(400 \mu \mathrm{g} / \mathrm{mL})$

(a)

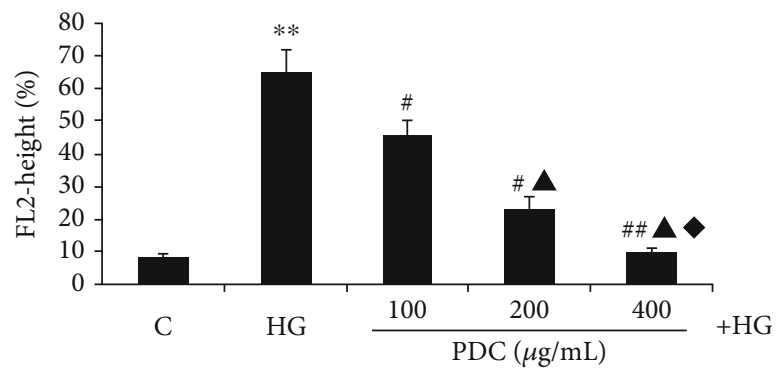

(b)

Figure 1: (a, b) Effect of PDC on calcium in HUVEC cells cultured in high glucose. Both D-glucose (33.3 mmol/L) and PDC (100, 200, and $400 \mu \mathrm{g} / \mathrm{mL}$ ) were incubated with HUVECs for 48 hours. Fluo-3 AM is a fluorescent probe for detecting calcium in cells by flow cytometry. C: control group; HG: high D-glucose group; PDC: Dendrobium candidum polysaccharide group. All data are expressed as $\bar{x} \pm \mathrm{S}(n=5)$. ${ }^{* *}$ $P<0.05$, compared with the control group; $\# P<0.05$, \#\#P<0.01 compared with the high glucose group; $\mathbf{\Delta} P<0.05$, compared with the high glucose + PDC $(100 \mu \mathrm{g} / \mathrm{mL})$ group; $>P<0.05$, compared with high glucose + PDC $(200 \mu \mathrm{g} / \mathrm{mL})$ group.

respectively), showing a concentration dependence $(P<0.05)$. The results show that PDC has a protective effect on protecting HUVECs from high glucose.

\subsection{Effect of PDC on Intracellular Calcium Concentration of} HUVEC Cells Cultured in High Glucose In Vitro. The results are shown in Figures 1(a) and 1(b). Compared with the control group, the intracellular calcium fluorescence intensity of HUVECs in the high glucose group significantly increased (the intracellular calcium ion fluorescence intensity was $12.15 \pm 1.78$ and $62.46 \pm 5.86$, respectively, $P<0.01$ ); compared with the high glucose group, the high glucose + PDC $(100,200$ and $400 \mu \mathrm{g} / \mathrm{mL})$ group significantly reduced the fluorescence intensity of calcium ions, in a concentrationdependent manner (intracellular calcium ion fluorescence intensity was $42.20 \pm 5.76,26.28 \pm 3.11$, and $11.49 \pm 2.48, P$ $<0.05)$. These indicate that PDC can prevent intracellular calcium overload caused by high glucose.
3.3. Effect of PDC on Reactive Oxygen Species and ROS in HUVEC Cells Cultured in High Glucose In Vitro. The results of flow cytometry were shown in Figures 2(a) and 2(b). Compared with the control group, the generation of reactive oxygen species in HUVEC cells in the high glucose group significantly increased (ROS fluorescence intensity was 4.41 \pm 0.96 and $14.00 \pm 1.64$, respectively, $P<0.05$ ); compared with the high glucose group, the levels of reactive oxygen species in the high glucose + PDC $(100,200$, and $400 \mu \mathrm{g} / \mathrm{mL})$ group significantly reduced (ROS fluorescence intensity was $6.14 \pm 0.83,5.83 \pm 0.86$, and 5.61 \pm 0.72 , respectively, $P<$ $0.05)$.

3.4. Effect of PDC on SOD in HUVEC Cells Cultured in High Glucose. As shown in Figure 3(a), compared with the control group, the intracellular SOD activity of the high glucose group significantly reduced (SOD activities were $66.14 \pm$ 1.70 and $24.02 \pm 1.67 \mathrm{U} / \mathrm{mg}$ protein, respectively, $P<0.05$ ). 

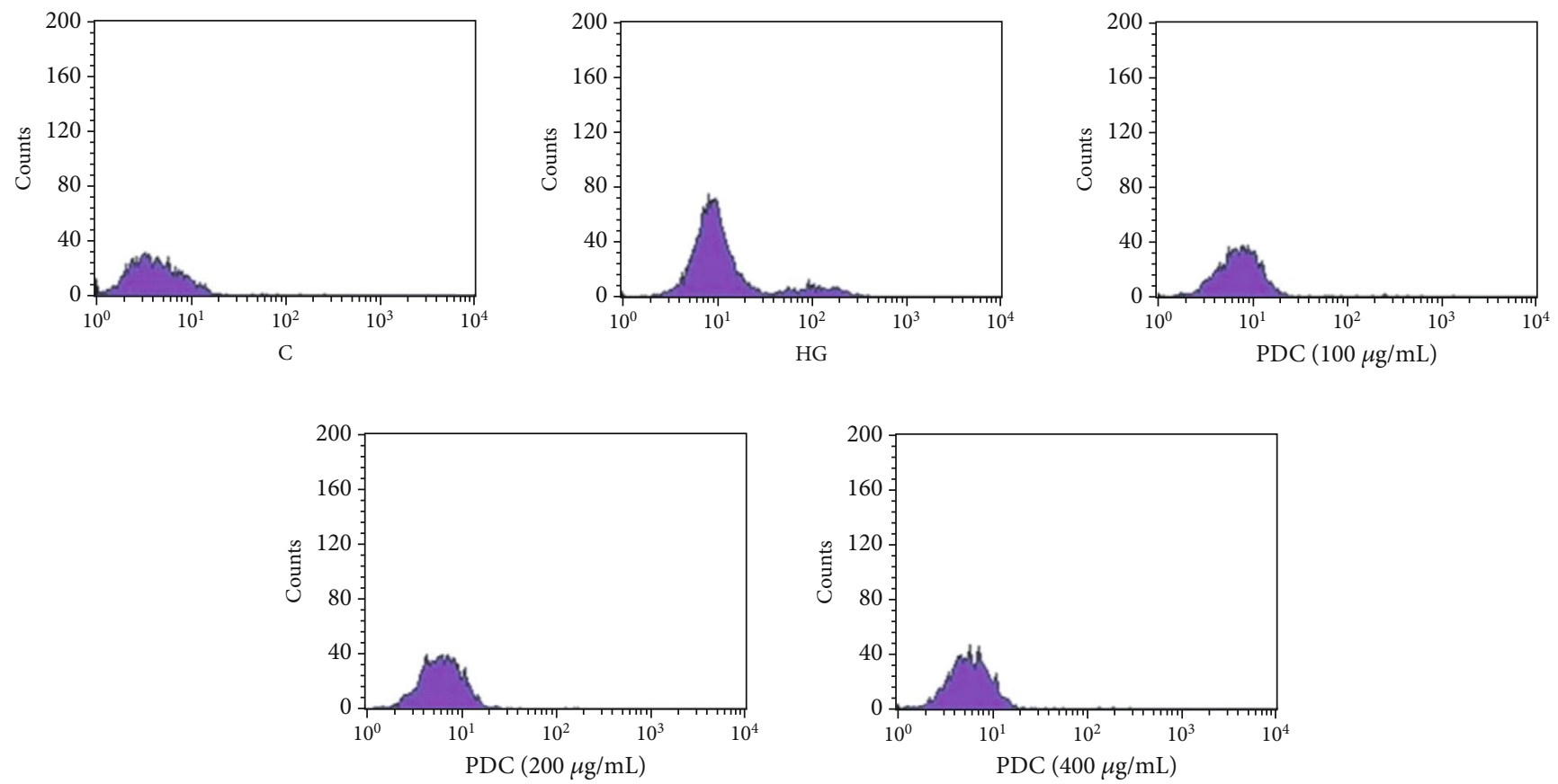

(a)

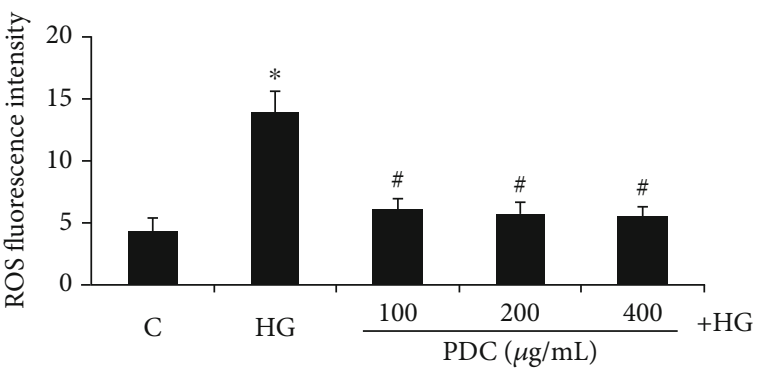

(b)

Figure 2: (a, b) Effect of PDC on ROS levels in HUVECs cultured in high glucose. Both D-glucose (33.3 mmol/L) and PDC (100, 200, and 400 $\mu \mathrm{g} / \mathrm{mL}$ ) were incubated with HUVECs for 48 hours. After staining with the fluorescent dye DCFH-DA, the intracellular ROS level was detected by flow cytometry. C: control group; HG: high D-glucose group; PDC: Dendrobium candidum polysaccharide group. All data are expressed as $\bar{x} \pm S(n=5) .{ }^{*} P<0.05$, compared with control group; $\# P<0.05$, compared with high glucose group.

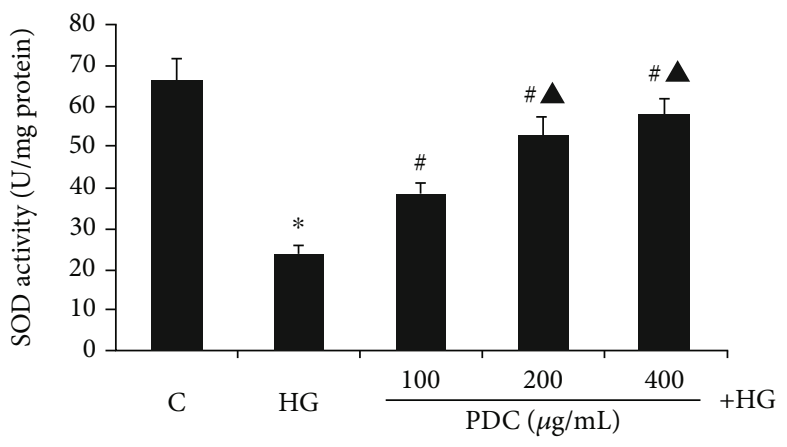

(a)

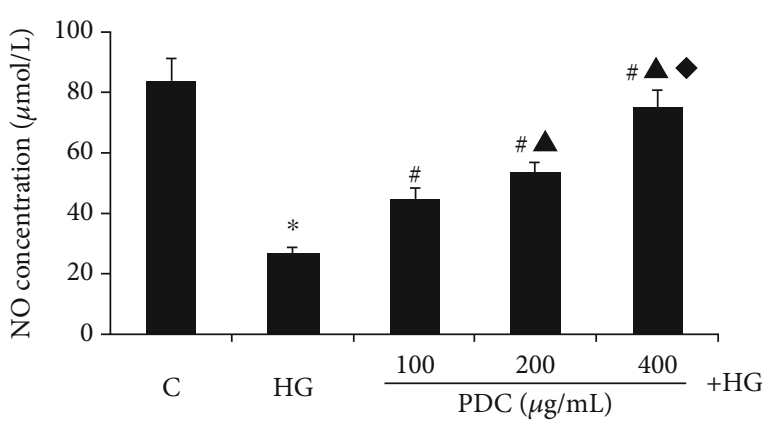

(b)

FIGURE 3: (a) Effect of PDC on SOD activity in HUVECs cells cultured in high glucose. (b) Effect of PDC on NO secretion from HUVECs cells cultured in high glucose. Both D-glucose $(33.3 \mathrm{mmol} / \mathrm{L})$ and PDC $(100,200$, and $400 \mu \mathrm{g} / \mathrm{mL})$ were incubated with HUVECs for 48 hours. Detection of SOD activity and NO secretion. C: control group; HG: high D-glucose group; PDC: Dendrobium candidum polysaccharide group. All data are expressed as $\overline{x \pm S}(n=5) .{ }^{*} P<0.05$, compared with the control group; $\# P<0.05$, compared with the high glucose group; $\mathbf{\Delta} P<0.05$, compared with the high glucose + PDC $(100 \mu \mathrm{g} / \mathrm{mL})$ group; $\diamond P<0.05$, compared with the high glucose + PDC $(200$ $\mu \mathrm{g} / \mathrm{mL})$ group. 
Compared with the high glucose group, the intracellular SOD activity in the high glucose $+\operatorname{PDC}(100,200$, and $400 \mu \mathrm{g} / \mathrm{mL})$ group significantly increased (SOD activities were $38.92 \pm$ $2.08,52.98 \pm 2.23$, and $58.29 \pm 2.67 \mathrm{U} / \mathrm{mg}$ protein, $P<0.05$ ). These results indicate that PDC can antagonize the reduction of the SOD expression in the human umbilical vein endothelial cell supernatant caused by in vitro cell culture under high glucose conditions.

\subsection{Effect of PDC on the Level of NO in Cultured HUVEC Cell} Culture Supernatant In Vitro. The results are shown in Figure 3(b). Compared with the control group, the NO secreted by HUVECs in the high glucose group significantly reduced ( $\mathrm{NO}$ concentrations in the culture medium were $83.90 \pm 7.19$ and $26.85 \pm 1.85 \mu \mathrm{mol} / \mathrm{L}, P<0.05)$. Compared with the high glucose group, the NO concentration in the cell culture fluid of the high glucose + PDC (100, 200, and 400 $\mu \mathrm{g} / \mathrm{mL}$ ) group significantly increased (the concentration of $\mathrm{NO}$ in the culture fluid was $44.58 \pm 3.87,53.73 \pm 3.12$, and $74.91 \pm 6.16 \mu \mathrm{mol} / \mathrm{L}, P<0.05)$.

\section{Discussion}

High glucose can aggravate the damage of IR and diabetic vascular endothelial cells [16]. It has been reported that the vascular endothelium-dependent vasodilation function decreases, blood flow velocity decreases, and the vasodilation function is negatively correlated with blood glucose concentration in normal subjects after glucose load, suggesting that high concentrations of glucose can induce the abnormality function of the vascular endothelial's cell. High glucose can cause vascular endothelial cell insufficiency in a variety of ways. The vascular endothelium is an important target for high glucose damage, and oxidative stress injury is an important factor for vascular endothelial damage $[17,18]$.

Numerous experiments have confirmed that both chronic and intermittent high glucose can increase oxidative stress and promote endothelial cell apoptosis through excessive production of ROS in the mitochondrial electron transport chain level $[16,19,20]$. These observations suggest that antioxidants can play a beneficial role in preventing endothelial cell damage caused by ROS. In this study, to evaluate the effect of PDC on the free radicals produced by endothelial cells caused by high glucose, we measured the content of SOD in the culture medium by colorimetry and the intracellular ROS level by flow cytometry, which is the most commonly used method of free radical generating ability. PDC is one of the main active ingredients of Dendrobium candidum and has good anti-inflammatory, antioxidant, and other biological activities [10, 21-23]. Different concentrations of PDC have been identified by Chen et al. to incresease the activity of SOD, which could contribute to inhibiting oxidative stress and apoptosis of human brain microvascular endothelial cells [24]. The results of this experimental study found that the intracellular ROS level increased significantly, and the activity of SOD in the culture medium decreased under high glucose culture, confirming once again the role of high glucose in oxidative damage and influencing endothelial cell proliferation during incubation with endothelial cells. The dose-dependent addition inhibited the production of ROS caused by high glucose, increased the activity of SOD, and significantly promoted endothelial cell proliferation. Therefore, it can be proved that PDC has a good active oxygen scavenging effect. This is consistent with the results of previous studies [25-27]. NO is a vasoactive substance synthesized by L-arginine as a substrate under the action of endothelial nitric oxide synthase in vascular endothelial cells. A reduction in NO can lead to an accelerated atherosclerotic process. A number of HUVECs in vitro culture experiments have confirmed that the level of NO decreases with the senescence of endothelial cells. In this experiment, we also observed that the NO level of HUVECs induced by high glucose decreased, which is consistent with the results of Hayashi et al. [28], and the experiment proved that PDC could significantly increase NO levels.

This study confirms that under the high glucose environment of $33.3 \mathrm{mmol} / \mathrm{L}$, the proliferation of human umbilical vein endothelial cells is suppressed, and the intracellular calcium concentration and the production of ROS increase, while the activity of SOD and NO production was reduced, but PDC can effectively antagonize the above changes in a dose-dependent manner. PDC can effectively antagonize the damage of HUVECs by high glucose culture, increase the activity of superoxide dismutase, and promote $\mathrm{NO}$ release. It is suggested that PDC may be a potential therapy pathway that is very important for protecting vascular endothelial cells. Its protective mechanism may be related to the antioxidant effect of PDC, which provides new ideas for the prevention and treatment of diabetes and vascular complications.

\section{Data Availability}

Data generated or analyzed during this study are included in this published article. The datasets generated during and/or analyzed during the current study are available from the corresponding author on reasonable request.

\section{Conflicts of Interest}

The authors declare that they have no competing interests.

\section{Authors' Contributions}

All authors participated in the experiments. Q.L., Y.L., and M.S. conceived the experiment(s). Y.L. and Z.C. conducted the experiment(s) and drafted the manuscript. Y.L. and Z.C. analyzed the results. All authors critically reviewed the manuscript. L.J. and Y.H. contributed in revision of the manuscript. All authors gave the final approval to the version submitted for publication.

\section{Acknowledgments}

We thank the grants from the Special Program of the Central Government to Guide Local Science and Technology Development, No. 2020YDDF0043, Philosophy and Social Science Planning Annual Project of Ningxia Hui Autonomous 
Region, No. 19NXAGL01, Key Research and Development Program of Ningxia Hui Autonomous Region in 2020, No. 2020beg03051, 2017 Project of Hunan Provincial Finance Department (Xiangcai Society refers to [2017] 61), Philosophy and Social Science Foundation of Hunan Province, No. 18YBA325, Project of Education Department of Hunan Province, No. 160546, Scientific Research Foundation of Hunan Provincial Health Commission, No. C2019055, Natural Science Foundation of Hunan Province in 2020, No. 2020JJ8012, and COVID-19 Special Project of Changsha Science and Technology Bureau, No. KQ2001019, supported by the Discipline Leader Project of "225" Project for High-level Health Talents Training in Hunan Province.

\section{References}

[1] Y. Dong, Y. Wu, H. C. Choi, and S. Wang, "Diabetic endothelium dysfunction, cardiovascular complications, and therapeutics," Journal Diabetes Research, vol. 2016, article 5349801, pp. 1-2, 2016.

[2] G. Orasanu and J. Plutzky, "The pathologic continuum of diabetic vascular disease," Journal of the American College of Cardiology, vol. 53, no. 5, pp. S35-S42, 2009.

[3] J.-X. Zhong, L. Liu, X.-Y. Sha, Y.-N. Wu, and M.-T. Chen, "Lycium barbarum polysaccharides protects retinal ganglion cells against oxidative stress injury," Neural Regeneration Research, vol. 15, no. 8, pp. 1526-1531, 2020.

[4] S. Shaw, X. Wang, H. Redd, G. D. Alexander, C. M. Isales, and M. B. Marrero, "High glucose augments the angiotensin IIinduced activation of JAK2 in vascular smooth muscle cells via the polyol pathway," The Journal of Biological Chemistry, vol. 278, no. 33, pp. 30634-30641, 2003.

[5] C. M. O. Volpe, P. H. Villar-Delfino, P. M. F. Dos Anjos, and J. A. Nogueira-Machado, "Cellular death, reactive oxygen species (ROS) and diabetic complications," Cell Death \& Disease, vol. 9, no. 2, pp. 119-119, 2018.

[6] E. Panieri and M. M. Santoro, "ROS signaling and redox biology in endothelial cells," Cellular and Molecular Life Sciences, vol. 72, no. 17, pp. 3281-3303, 2015.

[7] E. B. Peixoto and B. S. Pessoa, "antioxidant SOD mimetic prevents NADPH oxidase-induced oxidative stress and renal damage in the early stage of experimental diabetes and hypertension," American Journal of Nephrology, vol. 29, no. 4, pp. 309-318, 2009.

[8] S. D. M. Bandeira, G. D. S. Guedes, L. J. S. Da Fonseca et al., "Characterization of blood oxidative stress in type 2 diabetes mellitus patients: increase in lipid peroxidation and SOD activity," Oxidative Medicine and Cellular Longevity, vol. 2012, 13 pages, 2012.

[9] J. Liang, Y. Zeng, H. Wang, and W. Lou, "Extraction, purification and antioxidant activity of novel polysaccharides fromDendrobium officinaleby deep eutectic solvents," Natural Product Research, vol. 33, no. 22, pp. 3248-3253, 2019.

[10] Q. Li, J. Chen, Y. Li, T. Chen, J. Zou, and H. Wang, "Effect of polysaccharide of dendrobium candidum on proliferation and apoptosis of human corneal epithelial cells in high glucose," Medicine (Baltimore), vol. 96, no. 32, pp. e7773-e7773, 2017.

[11] H.-Y. Wang, Q.-M. Li, N.-J. Yu et al., "Dendrobium huoshanense polysaccharide regulates hepatic glucose homeostasis and pancreatic $\beta$-cell function in type 2 diabetic mice," Carbohydrate Polymers, vol. 211, pp. 39-48, 2019.

[12] H. S. Wu, "Studies on anti-hyperglycemic effect and its mechanism of Dendrobium candidum," Zhongguo Zhong Yao Za Zhi, vol. 29, no. 2, pp. 160-163, 2004.

[13] P. Kumar, A. Nagarajan, and P. D. Uchil, "Analysis of cell viability by the MTT assay," Cold Spring Harbor Protocols, vol. 2018, 2018.

[14] S. D'Alessandro, Y. Corbett, S. Parapini et al., "Malaria pigment accelerates MTT - formazan exocytosis in human endothelial cells," Parasitology, vol. 146, no. 3, pp. 399-406, 2019.

[15] Y. Li, Z. Cao, Q. Li, C. Wang, and Z. Zhou, "Effects of dendrobium polysaccharides on the functions of human skin fibroblasts and expression of matrix metalloproteinase-2 under high-glucose conditions," International Journal of Endocrinology, vol. 2021, Article ID 1092975, 8 pages, 2021.

[16] X. Wang, J. L. Martindale, Y. Liu, and N. J. Holbrook, "The cellular response to oxidative stress: influences of mitogenactivated protein kinase signalling pathways on cell survival," The Biochemical Journal, vol. 333, no. 2, pp. 291-300, 1998.

[17] L. Piconi, L. Quagliaro, R. Assaloni et al., "Constant and intermittent high glucose enhances endothelial cell apoptosis through mitochondrial superoxide overproduction," Diabetes/Metabolism Research and Reviews, vol. 22, no. 3, pp. 198203, 2006.

[18] M. A. Incalza, R. D'Oria, A. Natalicchio, S. Perrini, L. Laviola, and F. Giorgino, "Oxidative stress and reactive oxygen species in endothelial dysfunction associated with cardiovascular and metabolic diseases," Vascular Pharmacology, vol. 100, pp. 119, 2018.

[19] G. Basta, A. M. Schmidt, and R. De Caterina, "Advanced glycation end products and vascular inflammation: implications for accelerated atherosclerosis in diabetes," Cardiovascular Research, vol. 63, no. 4, pp. 582-592, 2004.

[20] T. Yokozawa, Y. A. Kim, H. Y. Kim, Y. A. Lee, and G.I. Nonaka, "Protective effect of persimmon peel polyphenol against high glucose-induced oxidative stress in LLC-PK(1) cells," Food and Chemical Toxicology, vol. 45, no. 10, pp. 1979-1987, 2007.

[21] J. A. Teixeira Da Silva and T. B. Ng, "The medicinal and pharmaceutical importance of Dendrobium species," Applied Microbiology and Biotechnology, vol. 101, no. 6, pp. 22272239, 2017.

[22] H. Chen, X. Li, Y. Xu et al., "Study on the polar extracts of Dendrobium nobile, D. officinale, D. loddigesii, and Flickingeria fimbriata: metabolite identification, content evaluation, and bioactivity assay," Molecules, vol. 23, 2018.

[23] X. Zhang, Y. Luo, G. Wei et al., "Physicochemical and antioxidant properties of the degradations of polysaccharides from Dendrobium officinale and their suitable molecular weight range on inducing HeLa cell apoptosis," Evidence-based Complementary and Alternative Medicine, vol. 2019, Article ID 4127360, 11 pages, 2019.

[24] L. Chen, S. Zhang, X. Liu, J. Dai, Z. Yan, and C. Wu, "Effects of dendrobium polysaccharides on human brain microvascular endothelial cell injury induced by ox-LDL via regulating the miR-378 expression," Cellular and Molecular Biology (Noisyle-Grand, France), vol. 66, no. 7, pp. 66-71, 2020.

[25] A. Luo, X. He, S. Zhou, Y. Fan, T. He, and Z. Chun, "In vitro antioxidant activities of a water-soluble polysaccharide derived from Dendrobium nobile Lindl. extracts," International 
Journal of Biological Macromolecules, vol. 45, no. 4, pp. 359363, 2009.

[26] Y. Liao, W.-Y. Yuan, W.-K. Zheng, A.-X. Luo, and Y.-J. Fan, "Correlation Between Functional Groups and Radical Scavenging Activities of Acidic Polysaccharides from Dendrobium," Zhong Yao Cai, vol. 38, no. 11, pp. 2281-2284, 2015.

[27] M. R. Paudel, M. B. Chand, B. Pant, and B. Pant, "Assessment of antioxidant and cytotoxic activities of extracts of Dendrobium crepidatum," Biomolecules, vol. 9, no. 9, p. 478, 2019.

[28] T. Hayashi, H. Matsui-Hirai, A. Miyazaki-Akita et al., "Endothelial cellular senescence is inhibited by nitric oxide: implications in atherosclerosis associated with menopause and diabetes," Proceedings of the National Academy of Sciences of the United States of America, vol. 103, no. 45, pp. 1701817023, 2006. 